\title{
Swipe kinematic differences in young children with autism spectrum disorders are task- and age-dependent: A smart tablet game approach
}

\author{
Szu-Ching Lu ${ }^{\text {a, }, \text { Philip Rowe }}{ }^{\mathrm{a}, \mathrm{b}}$, Christos Tachtatzis ${ }^{\mathrm{a}, \mathrm{c}}$, Ivan Andonovic ${ }^{\mathrm{a}, \mathrm{c}}$, Anna Anzulewicz ${ }^{\mathrm{d}}$, \\ Krzysztof Sobota ${ }^{\mathrm{e}}$, Jonathan Delafield-Butt ${ }^{\mathrm{a}}$ \\ ${ }^{a}$ Laboratory for Innovation in Autism, School of Education, University of Strathclyde, United Kingdom \\ ${ }^{\mathrm{b}}$ Department of Biomedical Engineering, University of Strathclyde, United Kingdom \\ ${ }^{\mathrm{c}}$ Department of Electronic and Electrical Engineering, University of Strathclyde, United Kingdom \\ ${ }^{\mathrm{d}}$ Faculty of Psychology, University of Warsaw, Poland \\ ${ }^{\mathrm{e}}$ Harimata Sp. z.o.o., Poland
}

\section{A R T I C L E I N F O}

\section{Keywords:}

Autism

Movement

Kinematics

Preschool

Motor development

Learning

Serious game assessment

\begin{abstract}
A B S T R A C T
The motor system is becoming increasingly recognized as an important site of disruption in autism spectrum disorders (ASD). However, the precise nature of this motor disruption remains unclear with some conflicting reports. We employed a smart tablet serious game approach, which did not require verbal instruction. Children's movements on the touch screen were recorded, and their kinematics computed from two games. One afforded goal-directed swipes, and the other free-style colouring. Children aged 25-79 months participated in this study, including 37 children with ASD and 45 typically developing (TD) children. Results revealed significant group, age, and task differences. In comparison to controls, children with ASD $<5$ years old performed faster goaldirected swipes, whereas those $\geq 5$ years old performed slower goal-directed swipes. In contrast, during freestyle drawing, children with ASD moved faster than the controls irrespective of age. Within the TD participants, the older subgroup ( $\geq 5$ years) performed faster movements than the younger subgroup ( $<5$ years) in both game contexts. However, the ASD older subgroup moved slower than their younger subgroup in the case of goaldirected swipes while no speed difference was observed in the case of free-style drawing. These findings reveal developmental differences in motor development in young children with ASD from their TD counterparts. Further, they demonstrate smart tablet gameplay can produce precise computational metrics of motor kinematics to characterize these differences deployable in schools, clinics and home settings for large-scale data collection for both research and clinical purposes that may ultimately enable accessible and scalable early detection of ASD.
\end{abstract}

\section{Introduction}

Disruption to movement in individuals with autism was first identified by Kanner, [1] but has received far less attention than its social or behavioural aspects. Recent studies demonstrate motor kinematics of purposeful movement are disrupted in autism in varied tasks, including horizontal arm movements [2], reaching [3], reach-and-grasping [4], reach-to-touch [5], and handwriting [6]. In each task a subtle, but significantly different action organization is employed by individuals with autism to complete it, in comparison to typical individuals. Gait [7-10], postural adjustments during gait [11], and postural adjustments during load-shift tasks [12] are also affected, and the efficient prospective organization of movements in a series, or action chain, is thwarted [13]. A landmark statistical meta-analysis across all available evidence concluded disruption to movement a pervasive feature across the autism spectrum and can be considered a core component of the syndrome [14]. The disruption to intentional movement and its affective engagement with others is a likely primary contributor to autism pathogenesis [15].

Yet, motor disruption is not included in the formal diagnostic criteria. Repetitive movements and behaviours are included in the diagnostic criteria in the United States [16] and worldwide [17], but the subtle neuromotor disturbance to purposeful behavior is not. Only 'clumsy and awkward' gait has so far been included in DSM-5 as an

\footnotetext{
* Corresponding author at: Laboratory for Innovation in Autism, University of Strathclyde, Graham Hills Building GH852, 40 George Street, Glasgow, G1 1QE, Scotland, United Kingdom

E-mail address: szuching.lu@strath.ac.uk (S.-C. Lu).
} 
associated symptom within the syndrome [16]. This lack of diagnostic inclusion is likely due to two outstanding questions. On the one hand, there is considerable debate whether or not disruption to the motor system extends across the spectrum, or is relevant only to a subset within it. And on the other, the precise nature of the motor disruption is still not well characterized, and so insufficiently defined for operational diagnostic criteria. In this paper, we address the character of the motor kinematics in young children with autism to add resolution to the latter.

New technologies in the domain of consumer smart devices and wearable sensors now offer improved, high-precision access to motor signature information, and coupled together with artificial intelligence data analytics of movement patterns may serve as possible early biomarker of the condition in young children [18-20], but the artificial intelligence methods usually deployed for diagnosis in these systems classify the movements in terms of complex 'black box' algorithms, rather than with conventional action kinematic variables which can be understood in the context of human neuromotor control principles that underpin psychological experience [21].

Of special interest has been upper limb action kinematics that carry out everyday tasks to manipulate objects and communicate in expressive gesture. Motor disturbance to a child's agency can alter the expression of everyday purposive behavior and thwart the capacity to efficiently enact an intention [22]. It can create difficulty in adaptive function and social engagement and consequent autistic social and emotional compensations $[15,23]$. It is therefore important to characterize this motor disturbance in terms of its action kinematics. Despite its critical role in manual object manipulation and gestural communication with others, the kinematic atypicalities of upper limb motor control in individuals with autism remain unclear, often with conflicting results between studies in relation to particular kinematic parameters such as movement speed [2,24].

Greater time-to-peak velocities during goal-directed arm movements were reported in individuals with autism compared to those in controls [25-27]. Longer deceleration times were also found [28], although the total percentage of time spent in deceleration was not significantly different from those in controls. Peak velocity during goal-directed movements appeared lower in young adults with autism [26] and in low-functioning children with autism [28] than those in controls, but this kinematic difference was inconsistent: it is absent in some reports $[24,25,29,30]$ and reversed in others [28]. Intelligence Quotient (IQ) and autism severity may play a role, for example Mari et al. (2003) reported high functioning children with autism move with greater peak velocity when reaching to grasp an object [28], and Forti et al. (2011) found a peak velocity positively correlated with IQ in their sample [29]. Moreover, peak velocity during an arm swing task was significantly higher in adults with autism than those in controls [2]. In all of these studies, motor kinematic differences are consistently reported, but their direction of difference differs between studies and group characteristics. There appears to be no simple, straight-forward kinematic characteristic difference in autism, yet upper limb kinematics by-and-large remain atypical.

A relatively consistent finding is that individuals with autism spectrum disorders (ASD) exhibit longer duration movements across a range of distances and target sizes [4,12,25,26,29-31], with the exception that Mari et al. (2003) found this feature only in low ability ASD children [28]; high ability ASD children exhibiting shorter movement durations. Similarly, Forti et al. (2011) reported an inverse relationship between IQ and movement duration [29], whereas movement duration was not found to be different between the typically developing (TD) and ASD groups in other studies [13,24]. Cook et al. (2013) found a shorter movement duration in ASD, however this was for a different task involving sinusoidal arm movements [2], rather than a task involving a specific spatial goal at its endpoint.

A major bottleneck in kinematic studies of individuals with autism, and especially children with autism, is the laboratory conditions under which they are normally conducted. Optical motion tracking technologies employed in the kinematic studies above require dedicated laboratories set within universities or clinical centres that require the child to come to these unfamiliar settings to take part. Further the tasks require didactic verbal instruction and cooperation on the part of the child. This can be difficult or impossible for some individuals with ASD, especially young children with limited linguistic comprehension or motivation for sharing the intentions of the experimenter. On the contrary, young children with or without ASD are generally attracted to tablet games and can easily engage without complicated instructions. Thus, we employed two smart tablet games to attract the interests of the children with minimal instructions. We reasoned this method would collect movement information from the children with greater ecological validity than our laboratory-based optical motion tracking paradigmatic counterparts.

Further, as we show above, the field of motor control in ASD requires clarification. Although the general finding of atypical motor kinematics has been reported across the literature, the precise nature of this atypicality is inconsistent. Thus, it is imperative more studies are carried out with greater numbers of subjects to improve statistical power and allow exploration of variance across the wide breadth of the autism spectrum, and especially over developmental time. Such large-scale data collection would be feasible with attractive games on mobile platforms that can be brought to the children in their daycare centres, classrooms, or clinics.

Further, previous kinematic studies of children focused on those aged 6-12 years. No study has yet focused on the motor kinematics of preschool children aged 2-6 years. Importantly, visual-motor development changes dynamically in early childhood, improving remarkably at 4-5 years old [32,33]. Also, the brainstem sensorimotor regions responsible for subsecond timing of adaptive movement develop differently from at least the preschool age onward in autism [34-37]. Thus, this study set out to determine the kinematic differences in young preschool children taking into account age level and task requirement.

For this paper, we employed smart tablet gameplay data from a previous study [18] (1) to compute how preschool children's motor kinematics differ between ASD and TD conditions, and by doing so (2) to test the feasibility of utilizing the smart tablet touch trajectory data for testing kinematic differences. Further, we sought to determine (3) age effects and (4) context-dependent effects on any kinematic differences between groups to advance a more precisely defined developmental characterization of kinematic differences in young children with ASD. These analyses extend the machine learning data science approach employed earlier [18], which presented 'black box' algorithms to classify the ASD and TD groups with high precision, to give more detailed and precise focus on the subsecond kinematics of goal-directed and free-style drawing and colouring in the two groups, at the different age levels analysed in this study.

\section{Methodology}

\subsection{Ethics approval}

The data used for analysis were from a previous project [18] carried in out in accordance with the Declaration of Helsinki and approved by the University of Strathclyde Ethics Committee. Prior to the study, written informed consent was obtained from the parents or legal guardians of the participants.

\subsection{Participants}

82 children were recruited in the study, including 37 children with ASD diagnosed according to ICD-10 criteria [17] and 45 TD children with no developmental concerns. There were 12 females in the ASD group while 13 females were in the TD group, and the age of the participants ranged from 25 to 79 months (Fig. 1). 


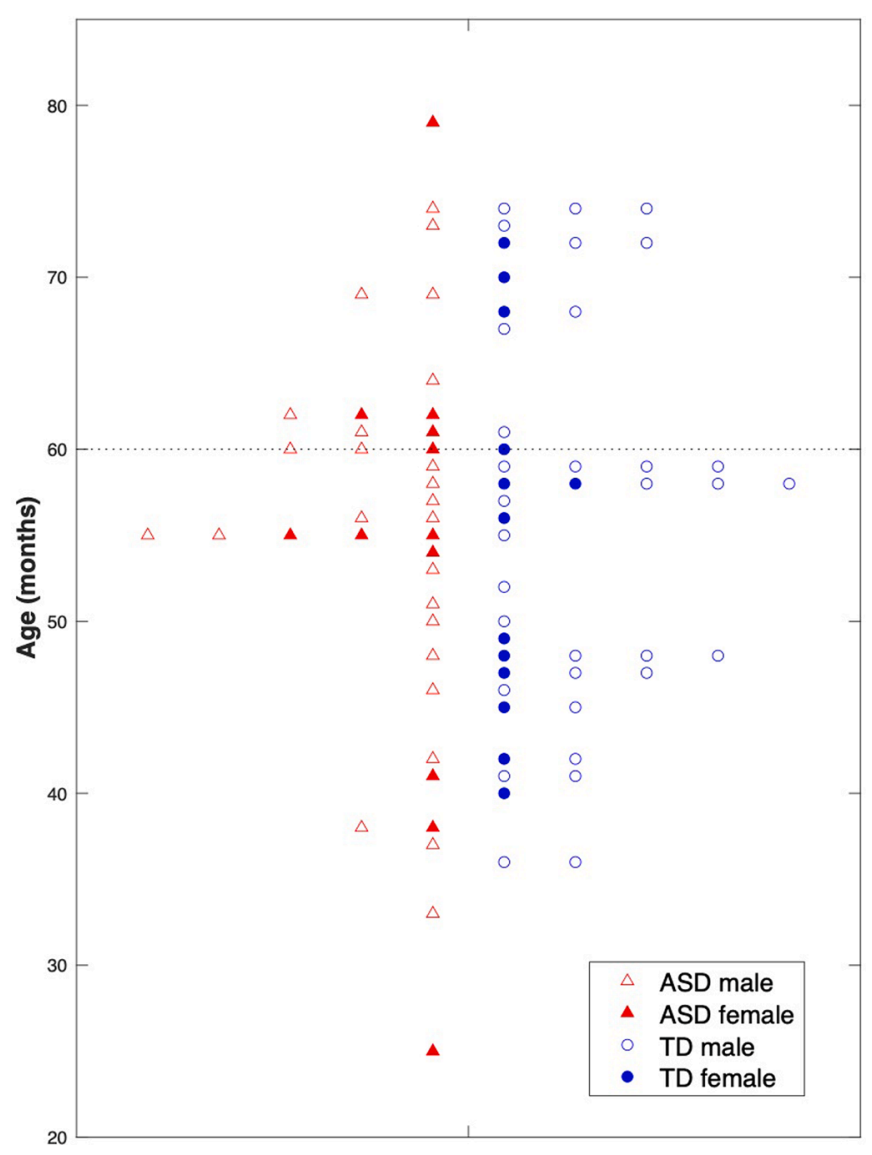

Fig. 1. Young children aged 25-79 months were included in this study, including 37 children with ASD and 45 TD children.

\subsection{Two smart tablet games}

Two smart tablet games, "Sharing" and "Creativity", were introduced to the participants on an iPad mini (Apple Inc.). The iPad was inserted in a bumper case and placed flat on a table during the gameplay. The gameplay was conducted in the school environment where the teacher and experimenter were presented. A 2-minute demonstration was provided before a 5 -minute play of each game. Minimal instructions were provided by the experimenter during the 2-minute demonstration while no intervention during the 5-minute play. The Sharing game was presented before the Creativity game. This smart tablet task requires visual ability and upper limb strength and stability. Participants played the games at their own pace and could pause or stop their engagement anytime during gameplay. Data collection continued throughout each 5minute session.

The Sharing game (Fig. 2a) offered a goal-directed context. A food item popped up on the screen after the Sharing game started. The food item would split into four pieces after the participant tapped on it, and then the participant could move the food pieces to the four animated characters. When a piece of food was released anywhere withing the target area, the food piece would be automatically allocated to a nearest plate. After each animated character had a piece of food, an animation of the characters eating the food would be presented and then the next food item would pop up. Participants played the game till the 5-minute
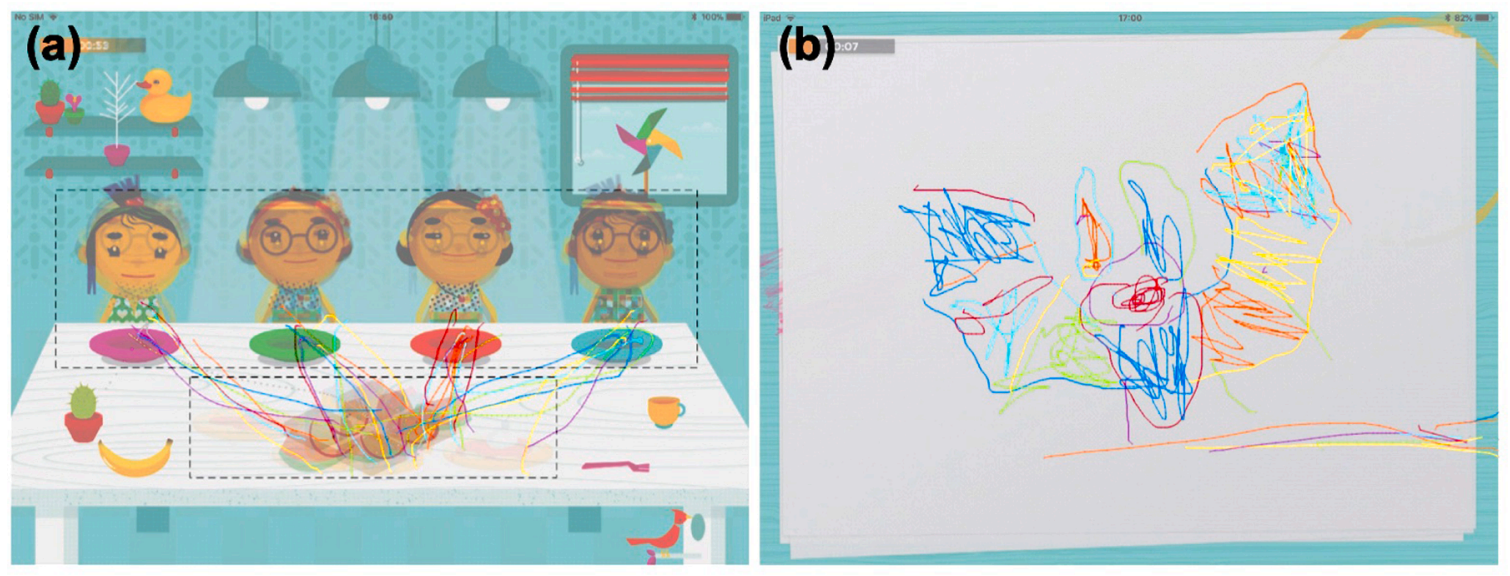

Fig. 2. Two sets of smart tablet gameplay were employed, including a Sharing game (a) and a Creativity game (b). For identifying the food-to-target swipes in the Sharing game, the food area (the bottom dashed box) and the target area (the top dashed box) were set when analysing the data. 
session ended.

The second game, Creativity (Fig. 2b), offered a free-style drawing context. The participant chose an object after the game started. Then, the participant traced the contours of the object while there was no requirement to trace the contours perfectly. Once the participant touched on the dashed contour lines, the contours of the object would be completed automatically. A color plate would pop up after the contours were completed, and the participant could choose from varied colours to do colouring. Participants could select another object to color at any time during the 5-minute session.

\subsection{Identify the swipe movements}

The timestamp, $\mathrm{x}$ - and $\mathrm{y}$-coordinates of the touch trajectories, and the touch phase were recorded at approximately $60 \mathrm{~Hz}$ during the gameplay while the data collection frequency varied slightly depending on the workload of the smart tablet. The touch phase described the interaction between the participant and the smart tablet and it was noted with integers (i.e., 0 : touch began; 1 : touch moved; 2 : stationary touch; 3: touch ended; 4: touch canceled). The x-coordinate value ranged from 0.5 to 1024 while the y-coordinate value ranged from 0.5 to 768 , and both with an increment of 0.5. With known screen size (i.e., 7.9 inch, or $200.66 \mathrm{~mm}$ ) and aspect ratio (i.e., 4:3) of the tablet, we could convert the recorded $\mathrm{x}$ - and $\mathrm{y}$-coordinates into $\mathrm{mm}(0.078 \mathrm{~mm}$ per pixel).

Customized MATLAB scripts were written for the data analysis. The touch phase information was used identify each swipe. A complete swipe movement was recorded with a start (touch phase ' 0 ') and an end (touch phase ' 3 ') of movement. Incomplete movements (i.e., movement segments recorded with a star and/or an end) or movements with a length of only two frames (i.e., a tap on the screen) were discarded. Movements involved with multiple finger touches were also removed from analysis. The procedure for the identification of complete swipes was the same for both Sharing and Creativity games while the goal-directed food sharing swipes were further selected using the 'food' and 'target' areas (Fig. 2a). The food area covered where different food items were presented on the screen, and the target area covered the plates and faces of the four animated characters.

\subsection{Swipe kinematics}

Fig. 3 presents an example of the food-to-target swipe. A total of five kinematic variables were computed for each swipe in the Sharing game:
(1) travelled distance: the cumulative distance between the consecutive touch points; (2) minimal distance: the direct distance between the start and end points; (3) distance difference: the difference between the travelled distance and the minimal difference; (4) duration: the length of time for each swipe; (5) average speed: the travelled distance divided by the duration.

For the Creativity game, three kinematic variables of each swipe were computed: (1) travelled distance; (2) duration; (3) average speed.

\subsection{Statistical analysis}

The non-normality of the data was indicated by the KolmogorovSmirnov test, and Mann-Whitney U tests were used to test for any kinematic differences between the ASD and TD swipes. The comparison between the ASD and TD swipes was first performed irrespective of age. As visuomotor abilities changes remarkably at $4-5$ years old $[32,33]$, we further compared the motor kinematics in two subgroups at ages $<5$ years and $\geq 5$ years. We expected the motor kinematics of the older subgroup ( $\geq 5$ years) with relatively matured visuomotor abilities would be similar to those observed in previous studies on 6-12 years old children. In contrast, different motor patterns may be observed in the younger subgroup ( $<5$ years) with less matured visuomotor abilities. In addition, we compared the motor kinematics between the two age levels within each group.

\section{Results}

The distributions of the kinematic variables are presented in Fig. 4, and the statistical results are demonstrated in Tables 1 and 2. For the kinematic variables, the duration and average speed demonstrated medium to large effect size $(r>0.30)$ and the distance difference showed small to medium effect size $(r>0.10)$. In addition, stronger effects $(r>0.30)$ were observed in the comparison between ASD and TD older subgroups, and the comparison between $<5$ and $\geq 5$ years within the TD group.

\subsection{Sharing game}

Irrespective of age levels, a total of 1585 food-to-target swipes were identified from the 37 children with ASD, and a total of 3201 were identified from the 45 TD children. The median travelled distance for the ASD group was $42.70 \mathrm{~mm}$, which was higher than the median of 41.78 $\mathrm{mm}$ for the TD group ( $p=0.034, r=0.03$ ). The median duration for the

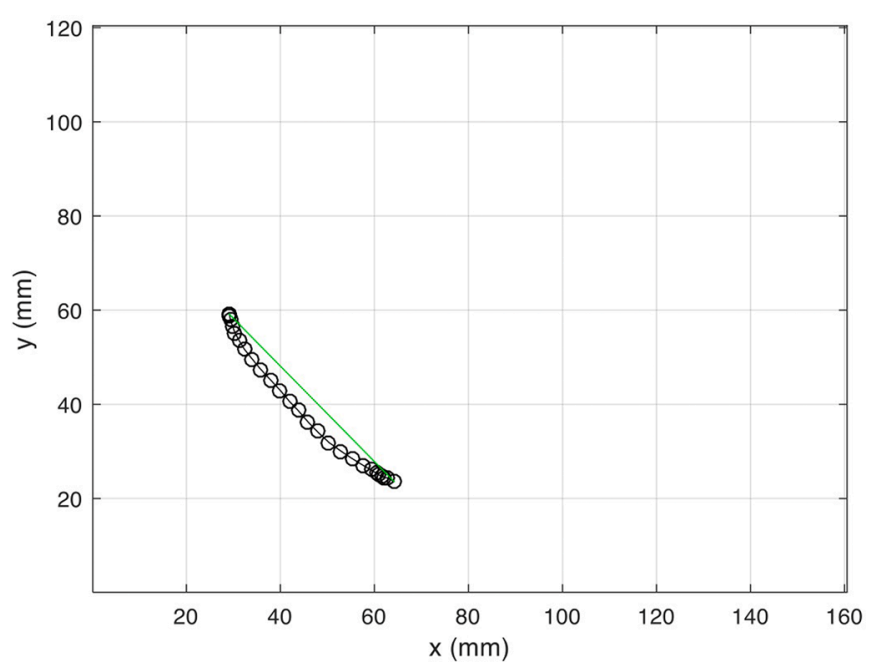

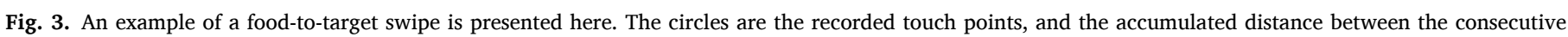

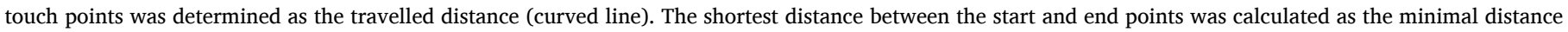
(straight line). 


\section{SHARING GAME}

Travelled Distance

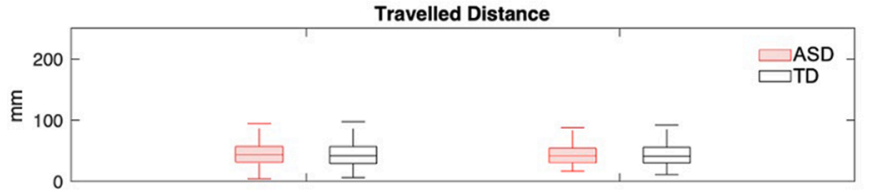

Duration

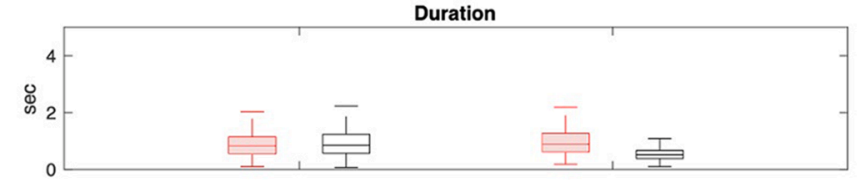

Average Speed

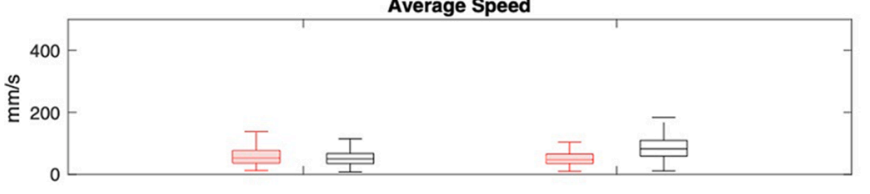

Minimal Distance
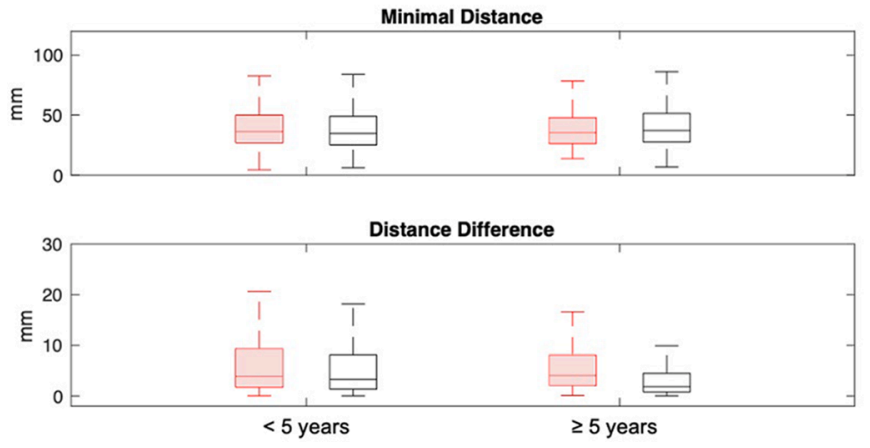

CREATIVITY GAME

Travelled Distance

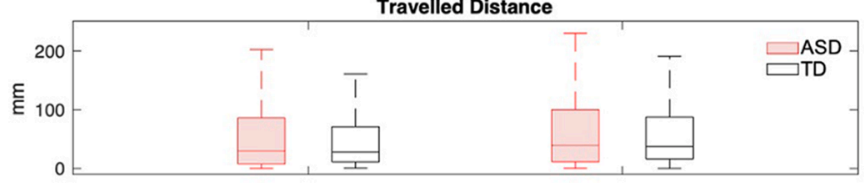

Duration

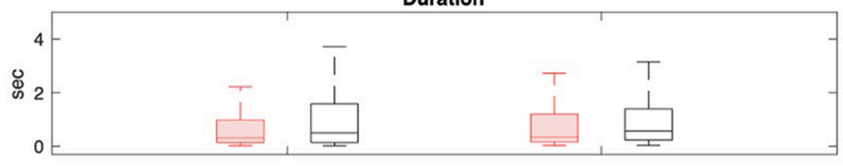

Average Speed

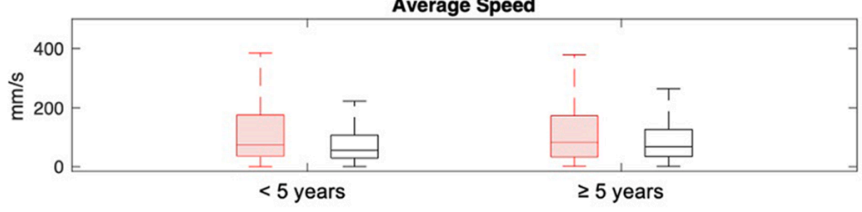

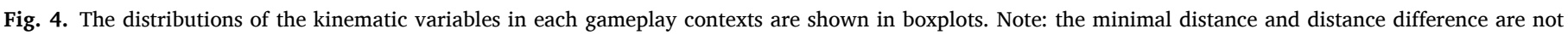
available in the Creativity game.

ASD group was $0.86 \mathrm{~s}$, which was greater than the median of $0.67 \mathrm{~s}$ for the TD group ( $p<0.001, r=0.15)$. The median average speed was $50.12 \mathrm{~mm} / \mathrm{s}$ for the ASD group, which was slower than the median of 58.88 for the TD group $(p<0.001, r=0.14)$. No significant difference in the minimal distance was found between the ASD $(\mathrm{Mdn}=35.92 \mathrm{~mm})$ and $\mathrm{TD}(\mathrm{Mdn}=35.73 \mathrm{~mm})$ groups. The median distance difference was 3.90 $\mathrm{mm}$ for the ASD group, which was greater than the median of $2.59 \mathrm{~mm}$ for the TD group $(p<0.001, r=0.14)$.

\subsubsection{Sharing game: TD vs. ASD at age $<5$ years}

In the younger subgroup ( $<5$ years), a total of 926 food-to-target swipes were identified from 23 children with ASD, and a total of 2008 food-to-target swipes were identified from 32 TD children. The median travelled distance for the ASD group was $43.51 \mathrm{~mm}$, which was higher than the median of $41.97 \mathrm{~mm}$ for the TD group ( $p=0.009, r=0.05)$. No significant difference in duration was found between the ASD $(\mathrm{Mdn}=0.83 \mathrm{~s})$ and TD $(\mathrm{Mdn}=0.85 \mathrm{~s})$ groups. The median average speed for the ASD group was $52.65 \mathrm{~mm} / \mathrm{s}$, which was faster than the median of $49.70 \mathrm{~mm} / \mathrm{s}$ for the TD group ( $p<0.001, r=0.03$ ). The median minimal distance for the ASD group was $36.18 \mathrm{~mm}$, which was greater than the median of $34.71 \mathrm{~mm}$ for the TD group ( $p=0.034, r=0.04)$. The median distance difference was $3.84 \mathrm{~mm}$ for the ASD group, which was greater than the median of $3.31 \mathrm{~mm}$ for the TD group ( $p=0.005, r=0.05)$.

\subsubsection{Sharing game: $T D$ vs. ASD at age $\geq 5$ years}

In the older subgroup ( $\geq 5$ years), a total of 659 food-to-target swipes were identified from 14 children with ASD, and a total of 1193 food-totarget swipes were identified from 13 TD children. No significant difference in the travelled distance was found between the ASD $(\mathrm{Mdn}=41.84 \mathrm{~mm})$ and $\mathrm{TD}(\mathrm{Mdn}=41.43 \mathrm{~mm})$ groups. The median duration was $0.88 \mathrm{~s}$ for the ASD group, which was greater than the median of $0.51 \mathrm{~s}$ for the TD group ( $p<0.001, r=0.46$ ). The median average speed was $46.82 \mathrm{~mm} / \mathrm{s}$ for the ASD group, which was slower than the median of $81.72 \mathrm{~mm} / \mathrm{s}$ for the TD group $(p<0.001, r=0.47)$. The median minimal distance was $35.38 \mathrm{~mm}$ for the ASD group, which was less than the median of $37.07 \mathrm{~mm}$ for the TD group $(p=0.004, r=$ 0.07). The median distance difference was $4.03 \mathrm{~mm}$ for the ASD group, which was greater than the median of $1.82 \mathrm{~mm}$ for the TD group ( $p<$ $0.001, r=0.29$ ).

\subsubsection{Sharing game: $T D<5$ years $v$. $T D \geq 5$ years}

Within the TD participants, some differences were observed between the two age subgroups. The median duration was $0.51 \mathrm{~s}$ for the TD older subgroup, which was less than the median of $0.85 \mathrm{~s}$ for the TD younger subgroup ( $p<0.001, r=0.42$ ). The median average speed was 81.72 $\mathrm{mm} / \mathrm{s}$ for the TD older subgroup, which was faster than the median of $49.70 \mathrm{~mm} / \mathrm{s}$ for the TD younger subgroup ( $p<0.001, r=0.45$ ). The median distance difference was $1.82 \mathrm{~mm}$ for the TD older subgroup, which was less than the median of $3.31 \mathrm{~mm}$ for the TD younger subgroup $(p<0.001, r=0.19)$.

\subsubsection{Sharing game: $A S D<5$ years $v$. $A S D \geq 5$ years}

The median duration was $0.88 \mathrm{~s}$ for the ASD older subgroup, which was greater than the median of $0.83 \mathrm{~s}$ for the ASD younger subgroup ( $p$ $=0.006, r=0.07)$. The median average speed was $46.82 \mathrm{~mm} / \mathrm{s}$ for the ASD older subgroup, which was slower than the median of $52.65 \mathrm{~mm} / \mathrm{s}$ for the ASD younger subgroup ( $p<0.001, r=0.45$ ). No significant difference in the distance difference was observed between the ASD older $(\mathrm{Mdn}=4.03 \mathrm{~mm})$ and younger $(\mathrm{Mdn}=3.84)$ subgroups. 
Table 1

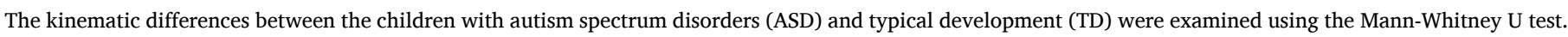

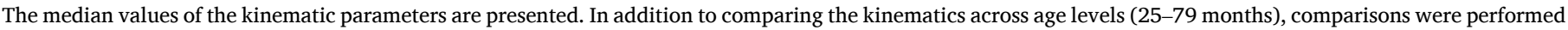
in the younger ( $<5$ years) and older ( $\geq 5$ years) subgroups.

\begin{tabular}{|c|c|c|c|c|c|c|c|c|c|}
\hline \multicolumn{5}{|c|}{ Sharing Game } & \multicolumn{5}{|c|}{ Creativity Game } \\
\hline All participants & ASD & TD & & & All participants & ASD & TD & & \\
\hline Number of participants & 37 & 45 & & & Number of participants & 37 & 45 & & \\
\hline Number of swipes & 1585 & 3201 & $\mathrm{p}$ value & Effect size r & Number of swipes & 3063 & 3551 & $\mathrm{p}$ value & Effect size $\mathrm{r}$ \\
\hline Travelled distance $(\mathrm{mm})$ & 42.70 & 41.78 & 0.034 & 0.03 & Travelled distance $(\mathrm{mm})$ & 33.51 & 30.58 & 0.916 & 0.00 \\
\hline Duration (sec) & 0.86 & 0.67 & $<0.001$ & 0.15 & Duration (sec) & 0.33 & 0.52 & $<0.001$ & 0.10 \\
\hline Average speed $(\mathrm{mm} / \mathrm{s})$ & 50.12 & 58.88 & $<0.001$ & 0.14 & Average speed $(\mathrm{mm} / \mathrm{s})$ & 77.11 & 58.90 & $<0.001$ & 0.13 \\
\hline Minimal distance (mm) & 35.92 & 35.73 & 0.978 & 0.00 & & & & & \\
\hline Distance difference $(\mathrm{mm})$ & 3.90 & 2.59 & $<0.001$ & 0.14 & & & & & \\
\hline Younger subgroup (<5yrs) & ASD & TD & & & Younger subgroup (<5yrs) & ASD & TD & & \\
\hline Number of participants & 23 & 32 & & & Number of participants & 23 & 32 & & \\
\hline Number of swipes & 926 & 2008 & $\mathrm{p}$ value & Effect size $r$ & Number of swipes & 1888 & 2491 & $\mathrm{p}$ value & Effect size $r$ \\
\hline Travelled distance $(\mathrm{mm})$ & 43.51 & 41.97 & 0.009 & 0.05 & Travelled distance $(\mathrm{mm})$ & 29.68 & 27.78 & 0.829 & 0.00 \\
\hline Duration $(\mathrm{sec})$ & 0.83 & 0.85 & 0.108 & 0.03 & Duration (sec) & 0.32 & 0.50 & $<0.001$ & 0.10 \\
\hline Average speed $(\mathrm{mm} / \mathrm{s})$ & 52.65 & 49.70 & $<0.001$ & 0.07 & Average speed $(\mathrm{mm} / \mathrm{s})$ & 73.98 & 55.45 & $<0.001$ & 0.15 \\
\hline Minimal distance (mm) & 36.18 & 34.71 & 0.034 & 0.04 & & & & & \\
\hline Distance difference $(\mathrm{mm})$ & 3.84 & 3.31 & 0.005 & 0.05 & & & & & \\
\hline Older subgroup ( $\geq$ 5yrs) & ASD & TD & & & Older subgroup ( $\geq 5 \mathrm{yrs}$ ) & ASD & TD & & \\
\hline Number of participants & 14 & 13 & & & Number of participants & 14 & 13 & & \\
\hline Number of swipes & 659 & 1193 & $\mathrm{p}$ value & Effect size $r$ & Number of swipes & 1175 & 1060 & $\mathrm{p}$ value & Effect size $r$ \\
\hline Travelled distance (mm) & 41.86 & 41.43 & 0.935 & 0.00 & Travelled distance $(\mathrm{mm})$ & 39.14 & 37.06 & 0.564 & 0.01 \\
\hline Duration (sec) & 0.88 & 0.51 & $<0.001$ & 0.46 & Duration (sec) & 0.35 & 0.58 & $<0.001$ & 0.12 \\
\hline Average speed (mm/s) & 46.82 & 81.72 & $<0.001$ & 0.47 & Average speed $(\mathrm{mm} / \mathrm{s})$ & 82.87 & 68.73 & $<0.001$ & 0.08 \\
\hline Minimal distance (mm) & 35.38 & 37.07 & 0.004 & 0.07 & & & & & \\
\hline Distance difference $(\mathrm{mm})$ & 4.03 & 1.82 & $<0.001$ & 0.29 & & & & & \\
\hline
\end{tabular}

Table 2

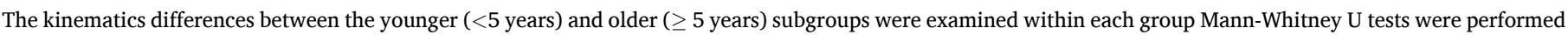
and the median kinematic values are presented. ASD: autism spectrum disorders; TD: typical development.

\begin{tabular}{|c|c|c|c|c|c|c|c|c|c|}
\hline \multicolumn{5}{|c|}{ ASD } & \multicolumn{5}{|c|}{ TD } \\
\hline Sharing Game & $<5$ yrs & $\geq 5 \mathrm{yrs}$ & & & Sharing Game & $<5$ yrs & $\geq 5 \mathrm{yrs}$ & & \\
\hline Number of participants & 23 & 14 & & & Number of participants & 32 & 13 & & \\
\hline Number of swipes & 926 & 659 & $\mathrm{p}$ value & Effect size $r$ & Number of swipes & 2008 & 1193 & $\mathrm{p}$ value & Effect size $r$ \\
\hline Travelled distance (mm) & 43.51 & 41.86 & 0.081 & 0.04 & Travelled distance (mm) & 41.97 & 41.43 & 0.519 & 0.01 \\
\hline Duration (sec) & 0.83 & 0.88 & 0.006 & 0.07 & Duration $(\mathrm{sec})$ & 0.85 & 0.51 & $<0.001$ & 0.42 \\
\hline Average speed (mm/s) & 52.65 & 46.82 & $<0.001$ & 0.09 & Average speed $(\mathrm{mm} / \mathrm{s})$ & 49.70 & 81.72 & $<0.001$ & 0.45 \\
\hline Minimal distance $(\mathrm{mm})$ & 36.18 & 35.38 & 0.230 & 0.03 & Minimal distance $(\mathrm{mm})$ & 34.71 & 37.07 & $<0.001$ & 0.08 \\
\hline Distance difference (mm) & 3.84 & 4.03 & 0.469 & 0.02 & Distance difference (mm) & 3.31 & 1.82 & $<0.001$ & 0.19 \\
\hline Creativity Game & $<5$ yrs & $\geq 5 \mathrm{yrs}$ & & & Creativity Game & $<5$ yrs & $\geq 5 \mathrm{yrs}$ & & \\
\hline Number of participants & 23 & 14 & & & Number of participants & 32 & 13 & & \\
\hline Number of swipes & 1888 & 1175 & $\mathrm{p}$ value & Effect size $r$ & Number of swipes & 2491 & 1060 & $\mathrm{p}$ value & Effect size r \\
\hline Travelled distance (mm) & 29.68 & 39.14 & 0.003 & 0.05 & Travelled distance (mm) & 27.78 & 37.06 & $<0.001$ & 0.09 \\
\hline Duration (sec) & 0.32 & 0.35 & 0.022 & 0.04 & Duration $(\mathrm{sec})$ & 0.50 & 0.58 & 0.007 & 0.05 \\
\hline Average speed $(\mathrm{mm} / \mathrm{s})$ & 73.98 & 82.87 & 0.872 & 0.00 & Average speed $(\mathrm{mm} / \mathrm{s})$ & 55.45 & 68.73 & $<0.001$ & 0.08 \\
\hline
\end{tabular}

\subsubsection{Sharing game: summary}

The ASD older subgroup ( $\geq 5$ years) moved slower than the controls when completing goal-directed swipes while the ASD younger subgroup $(<5$ years) moved faster than the controls. In both age subgroups, children with ASD deviated more from the minimal distance than the controls when performing goal-directed swipes. Within the TD participants, the older subgroup performed faster and less deviated goaldirected swipes than the younger subgroup. On the contrary, the ASD older subgroup moved slower than the ASD younger subgroup when completing the goal-directed swipes with no difference in the deviation from the minimal distance.

\subsection{Creativity game}

Irrespective of age levels, a total of 3063 free-style drawing swipes were identified from the 37 children with ASD, and a total of 3551 swipes were identified from the $45 \mathrm{TD}$ children. No significant difference was observed between the ASD $(\mathrm{Mdn}=33.51 \mathrm{~mm})$ and $\mathrm{TD}(\mathrm{Mdn}=30.58$ $\mathrm{mm}$ ) groups. The median duration was $0.33 \mathrm{~s}$ for the ASD group, which was less than the median of $0.52 \mathrm{~s}$ for the TD group $(p<0.001, r=0.10)$. The median average speed was $77.11 \mathrm{~mm} / \mathrm{s}$ for the ASD group, which was faster than the median of $58.90 \mathrm{~mm} / \mathrm{s}$ for the TD group $(p<0.001, r$ $=0.13$ ).

\subsubsection{Creativity game: $T D$ vs. ASD at age $<5$ years}

In the younger subgroup ( $<5$ years), a total of 1888 free-style drawing swipes were identified from 23 children with ASD, and a total of 2491 swipes were identified from 32 TD children. Similar to what we found in the comparison irrespective of age levels, no significant difference was observed between the ASD $(\mathrm{Mdn}=29.68 \mathrm{~mm})$ and 
$\mathrm{TD}(\mathrm{Mdn}=27.78 \mathrm{~mm})$ groups. The median duration was $0.32 \mathrm{~s}$ for the ASD group, which was less than the median of $0.50 \mathrm{~s}$ for the TD group ( $p$ $<0.001, r=0.10$ ). The median average speed was $73.98 \mathrm{~mm} / \mathrm{s}$ for the ASD group, which was faster than the median of $55.45 \mathrm{~mm} / \mathrm{s}$ for the TD group $(p<0.001, r=0.15)$.

\subsubsection{Creativity game: $T D v s$. ASD at age $\geq 5$ years}

In the older subgroup ( $\geq 5$ years), a total of 1175 free-style drawing swipes were identified from 14 children with ASD, and a total of 1060 swipes were identified from 13 TD children. Again, no significant difference was observed between the ASD ( $\mathrm{Mdn}=39.14 \mathrm{~mm}$ ) and TD $(\mathrm{Mdn}=37.06 \mathrm{~mm})$ groups. The median duration was $0.35 \mathrm{~s}$ for the ASD group, which was less than the median of $0.58 \mathrm{~s}$ for the TD group ( $p<$ $0.001, r=0.12$ ). The median average speed was $82.87 \mathrm{~mm} / \mathrm{s}$ for the ASD group, which was faster than the median of $68.73 \mathrm{~mm} / \mathrm{s}$ for the TD group $(p<0.001, r=0.08)$.

\subsubsection{Creativity game: $T D<5$ years $v s . T D \geq 5$ years}

Within the TD participants, the median average speed was 68.73 $\mathrm{mm} / \mathrm{s}$ for the older subgroup, which was faster than the median of 55.45 $\mathrm{mm} / \mathrm{s}$ for the younger subgroup ( $p<0.001, r=0.08$ ).

\subsubsection{Creativity game: $A S D<5$ years $v s$. $A S D \geq 5$ years}

No significant difference in the average speed was observed between the ASD older $(\mathrm{Mdn}=82.87 \mathrm{~mm} / \mathrm{s})$ and younger $(\mathrm{Mdn}=73.98 \mathrm{~mm} / \mathrm{s})$ subgroups.

\subsubsection{Creativity game: summary}

Children with ASD performed faster free-style drawing swipes than the controls irrespective of age levels. The TD older subgroup ( $\geq 5$ years) performed faster free-style drawing swipes in comparison to their younger subgroup $(<5$ years), however, no speed difference was observed between the ASD older and younger subgroups.

\section{Discussion}

This study examined the kinematic differences between children with ASD and TD. Further, as the visuomotor development was reported to change rapidly at 4-5 years old [32,33], we examined differences in younger ( $<5$ years) and older ( $\geq 5$ years) subgroups with their different levels of visuomotor ability. Given most previous studies on the motor kinematics in children with ASD examined participants 6-12 years old, we expected to see similar results in the older subgroup in our study.

Two sets of smart tablet gameplay were used, the Sharing game that involved goal-directed swipes and the Creativity game that involved free-style drawing and colouring gestures with no requirement for particular point-to-point goal-directedness. Different motor kinematics were observed in these different game contexts. The ASD younger subgroup ( $<5$ years) performed faster goal-directed swipes than the controls during the Sharing game, whereas the ASD older subgroup $(\geq 5$ years) performed slower goal-directed swipes than the controls. In addition to the differences in movement speed, children with ASD deviated more from the minimal food-to-target distance than the controls irrespective of age. In the Creativity game, children with ASD moved faster than the controls in both age subgroups when performing free-style drawing gestures. However, it should be noted that the effect size was minimal $(r<0.10)$ when comparing the ASD and TD younger subgroups while stronger effect $(r>0.30)$ was observed in the older subgroup comparison. The larger effect size $(r>0.30)$ in the older subgroup comparison appears to indicate developmental stasis in ASD in this age range.

\subsection{Goal-directed movements}

The slower goal-directed swipes in the ASD older subgroup ( $\geq 5$ years) in our study were consistent with previous reports on reach-to- grasp movements that reported longer movement durations in individuals with ASD in comparison to controls [4,25,30], with an exception in one study that did not find this difference in children with IQ score $\geq 80$ in the Weschler Intelligence Scale for Children [28]. The majority of the participants in these reach-to-grasp studies were over 5 years old, with very few children under 5 years old. In the reach-to-grasp experiments by Campione et al. (2016), the kinematics of participants 4.3 to 5.9 years old were reported, but without an investigation of the age effect [25]. These reach-to-grasp studies also used a fixed target for the point-to-point activities, whereas in our study the start and end points of the food-to-plate swipes could vary within a small area. Despite the differences in design, our results agree with these point-to-point studies in the movement speed that the ASD older subgroup $(\geq 5$ years) moved slower than the controls when performing food-to-target swipes.

With our food-to-target swipe data, we also calculated the difference between the minimal distance and the cumulative travelled distance, which can reflect the efficiency of a movement. Participants with ASD demonstrated greater distance differences than the controls, irrespective of age, which suggests less efficient movement in the ASD group when completing a goal-directed task. Within the TD participants, the distance difference was significantly smaller in the older subgroup ( $\geq 5$ years), indicating less deviation from the minimal distance suggestive of motor learning. However, no significant change in the distance difference was observed between the ASD older and younger subgroups, which suggests this movement strategy does not change during this age range. This appears to reflect a stagnation in motor learning or motor skill improvement in the ASD group between 3- and 6-years age.

Our findings in the food-to-target swipes are also in line with some of other point-to-point studies that longer movement durations were observed in participants with ASD in comparison to the controls [13,26, 27] except for those with IQ scores higher than 70 [38]. Forti et al. (2011) observed longer movement durations in young children with ASD aged around 3.5 years [29], which is opposite to what we found in our study. However, the task Forti et al. used in their study is considered more complex than ours, in which their young participants were instructed to transport a ball and drop it into a hole [29].

\subsection{Free-style movements}

Different from the food-to-target tasks available in the Sharing game, the Creativity game provided fewer visual cues for stopping or starting a movement, or guiding its direction. In this relatively unconstrained game context, we observed faster free-style drawing gestures in children with ASD when compared to controls, irrespective of age. And their speed did not change between ages. This is in contrast to the TD children whose speed increased significantly in the older age group. Again, similar to the Sharing game above, there appears a lack of developmental change in the motor characteristics of the gameplay between the two age groups in ASD.

In a previous arm swing study, faster and jerkier movements in adults with ASD in comparison to controls were reported [2]. Similarly, in a reach-to-point task arm movements were found to be faster and jerkier [5]. Our data could agree with the notion that individuals with ASD have difficulties sensing and modulating the moment-by-moment point in space of an action vis-à-vis its goal. The faster swipes in ASD in the absence of clear start and end points in our Creativity game could compensate for these motor modulation difficulties. It is especially interesting to note that the scale of movement in the smart tablet Creativity game and whole arm movements are very different tasks, but nonetheless require comparable motor control fundamentals.

\subsection{Limitations and future considerations}

There are some limitations to this study and some future considerations to be noted. We employed smart tablet games in our study to 
facilitate data collection in a fun and enjoyable manner, and to present more ecologically valid motor measurements. However, the participants' previous experience in using such a device may affect their performance. To mitigate this possible experience-dependent effect, a 2minute demonstration and practice session preceded the 5-minute data collection phase for each game. This allowed all participants to become familiar with the game with the aim to reduce experiencedependent differences. Further, in this study the Sharing game was always presented first. Fatigue over time could affect the results, especially in the Creativity game.

The smart tablet gameplay paradigm allowed an ecological approach for the collection of movement data. However, the sensory feedback provided by our smart tablet tasks is different from those in other reachto-grasp studies. A reduction in somatosensory integration in ASD during a force control activity has been reported [39]. The impact of differences in sensory feedback on motor performance should be noted and considered in subsequent work.

Intelligence level is reported to be related to the movement kinematics $[28,38]$. However, our study did not include IQ nor other cognitive assessment scores, which prevented us from investigating the effect of this factor on their swipe kinematics. The inclusion of IQ scores in future studies will be beneficial for a comprehensive understanding of the motor signatures in ASD. The lack of a standardized severity measure of ASD symptoms in this study prevented correlation between the motor disturbance and ASD symptom severity, which may be considered for future research to give additional insight. Lastly, body size of the participants was not noted in this study. The length of upper limb and finger size could affect motor metrics $[5,40]$.

It is worth noting that in comparison to point-to-point tasks employed in previous studies [4,13,24-26,26,28-30,38], there were no precise start and end points in our Sharing game. The piece of food was automatically allocated to the nearest plate if it was released within the target area (Fig. 2a). Participants learnt about this game design during the 2-minute demonstration and may release the food piece away from the plate on purpose, which made it unfair to consider those movements away from the plate as 'inaccurate' movements. However, the goal-directed task in our Sharing game tablet paradigm and goal-directed tasks employed by other teams show a comparable characteristics of slower movements with longer durations in ASD [4,13, 25-27,30]. A complementary set of precise point-to-point tasks on the smart tablet could be included in future studies to provide an in-depth comparison of the neuromotor control patterns between these paradigms. A modified game design with a precise ending point will also allow the study of movement accuracy and consistency, and their relationships with the movement speed. One solution has been proposed in other work from our lab [41]. Finally, although we observed significant differences between the two age subgroups, it should be noted that this was a cross-sectional, rather than a longitudinal study. A future longitudinal study that employs the same paradigm will give further resolution to understanding motor development in this age range.

\subsection{Aetiology of the ASD motor disruption}

Action kinematics are psychomotor expressions of underlying neuromotor physiology. There is likely to be a primary disruption in the neural systems that underpin these movements, the measured action kinematics a manifestation of those neural disruptions. Therefore, kinematic studies that address fundamental motor control theory, such as feed-forward and feed-back systems in motor control [31], prospective motor control such as shown for infant movement [42-44], predictive coding in motor systems [45,46] or entropy analyses [47] will begin to afford insight into disruptions of those neural systems in ASD, affording significant insight.

One possible underlying disruption is in the integration of sensory and motor information at the level of the brainstem, especially involving the inferior olivary nucleus [35]. This is supported by basic upper limb motor kinematics that show increased jerk amplitude at around $13 \mathrm{~Hz}$ [2] that is a predicted neurophysiological effect of disruption to the inferior olive [48-50] and evidence of volumetric and morphometric differences in growth of brainstem sensorimotor regions [34]. In this study, we have shown that motor kinematics maybe a valuable marker for ASD, indicative of underlying disruption to brainstem-cerebellar sensorimotor integration.

\subsection{Implications for clinical, educational, and parental practice}

This study adds to a growing body of evidence to demonstrate a fundamental disturbance to motor control in young children with autism. This evidence helps to raise awareness of the motor differences in children with autism as fundamental to their psychomotor condition, providing improved understanding that can inform a social perception of these motor differences, and enable improved learning support and accommodation in clinical, educational, and home settings.

\subsection{Conclusions}

In sum, we show that motor kinematic differences in preschool children with autism are age- and task-dependent. Importantly, we reveal evidence of a developmental stasis, or lack of developmental change in motor kinematics between 3 and 6 years of age in autism, important for understanding psychological development and learning, as well as neuromotor development. This study further demonstrates the practical benefit of serious game paradigms on smart tablet devices to investigate the motor metrics of young children. Where other smart tablet paradigms may be available for recording human kinematics [51, 52], these are not gamified and require verbal instruction that can be difficult for children with autism to follow. We demonstrated the advantage of using an attractive smart tablet gameplay approach for efficient and precise kinematic data collection with young children. This smart tablet gameplay paradigm can be employed in schools, clinics, or homes to assist large-scale data collection for both research and clinical purposes, and ultimately can underpin accessible, scalable early detection of ASD in young children [53].

\section{Declaration of Competing Interest}

The authors declare the following financial interests/personal relationships which may be considered as potential competing interests: Anna Anzulewicz and Krzysztof Sobota were employed by Harimata Sp. z.o.o. with vesting option agreements. Szu-Ching Lu, Philip Rowe, Christos Tachtatzis, Ivan Andonovic, and Jonathan Delafield-Butt declare no competing interests.

\section{Supplementary materials}

Supplementary material associated with this article can be found, in the online version, at doi:10.1016/j.dscb.2022.100032.

\section{References}

[1] L. Kanner, Autistic disturbances of affective contact, Nerv. Child 2 (1943) 217-250.

[2] J.L. Cook, S.-.J. Blakemore, C. Press, Atypical basic movement kinematics in autism spectrum conditions, Brain 136 (2013) 2816-2824.

[3] L.-A.R. Sacrey, T. Germani, S.E. Bryson, L. Zwaigenbaum, Reaching and grasping in autism spectrum disorder: a review of recent literature, Front. Neurol. 5 (2014) 6.

[4] A.M.B. Stoit, H.T. van Schie, D.I.E. Slaats-Willemse, J.K. Buitelaar, Grasping motor impairments in autism: not action planning but movement execution is deficient, J. Autism Dev. Disord. 43 (2013) 2793-2806.

[5] E.B. Torres, et al., Autism: the micro-movement perspective, Front. Integr. Neurosci. 7 (2013).

[6] A. Kushki, T. Chau, E. Anagnostou, Handwriting difficulties in children with autism spectrum disorders: a scoping review, J. Autism Dev. Disord. 41 (2011) 1706-1716. 
[7] E. Biffi, et al., Gait pattern and motor performance during discrete gait perturbation in children with autism spectrum disorders, Front. Psychol. 9 (2018) 2530.

[8] J.S. Dufek, J.D. Eggleston, J.R. Harry, R.A Hickman, A comparative evaluation of gait between children with autism and typically developing matched controls, Med. Sci. Basel Switz. 5 (2017).

[9] B.-.O. Lim, D. O'Sullivan, B.-.G. Choi, M.-.Y Kim, Comparative gait analysis between children with autism and age-matched controls: analysis with temporalspatial and foot pressure variables, J. Phys. Ther. Sci. 28 (2016) 286-292.

[10] O. Manicolo, M. Brotzmann, P. Hagmann-von Arx, A. Grob, P. Weber, Gait in children with infantile/atypical autism: age-dependent decrease in gait variability and associations with motor skills, Eur. J. Paediatr. Neurol. EJPN Off. J. Eur. Paediatr. Neurol. Soc. 23 (2019) 117-125.

[11] N.J. Rinehart, et al., Gait function in high-functioning autism and Asperger's disorder : evidence for basal-ganglia and cerebellar involvement? Eur. Child Adolesc. Psychiatry 15 (2006) 256-264.

[12] C. Schmitz, J. Martineau, C. Barthélémy, C. Assaiante, Motor control and children with autism: deficit of anticipatory function? Neurosci. Lett. 348 (2003) 17-20.

[13] M. Fabbri-Destro, L. Cattaneo, S. Boria, G Rizzolatti, Planning actions in autism, Exp. Brain Res. 192 (2009) 521-525.

[14] K.A. Fournier, C.J. Hass, S.K. Naik, N. Lodha, J.H. Cauraugh, Motor coordination in autism spectrum disorders: a synthesis and meta-analysis, J. Autism Dev. Disord. 40 (2010) 1227-1240.

[15] C. Trevarthen, J.T. Delafield-Butt, Autism as a developmental disorder in intentional movement and affective engagement, Front. Integr. Neurosci. 7 (2013).

[16] American Psychiatric Association. Diagnostic and statistical manual of mental disorders (DSM-5®). Am. Psychiatric Pub, 2013).

[17] World Health Organization, The ICD-10 classification of mental and behavioural disorders: clinical descriptions and diagnostic guidelines, World Health Organ. (1992).

[18] A. Anzulewicz, K. Sobota, J.T. Delafield-Butt, Toward the autism motor signature: gesture patterns during smart tablet gameplay identify children with autism, Sci. Rep. 6 (2016) 31107.

[19] A. Crippa, et al., Use of machine learning to identify children with autism and their motor abnormalities, J. Autism Dev. Disord. 45 (2015) 2146-2156.

[20] D.R. Hocking, K. Caeyenberghs, What is the Nature of motor impairments in autism, are they diagnostically useful, and what are the implications for intervention? Curr. Dev. Disord. Rep. 2 (2017) 19-27.

[21] J. Delafield-But, Autism and Panpsychism: putting process in mind, J. Conscious. Stud. 28 (2021) 76-90.

[22] J.T. Delafield-Butt, N.Sensorimotor intentionality Gangopadhyay, The origins of intentionality in prospective agent action, Dev. Rev. 33 (2013) 399-425.

[23] J. Cook, From movement kinematics to social cognition: the case of autism, Philos. Trans. R. Soc. B Biol. Sci. 371 (2016), 20150372.

[24] A.M. Dowd, J.L. McGinley, J.R. Taffe, N.J Rinehart, Do planning and visual integration difficulties underpin motor dysfunction in autism? A kinematic study of young children with autism, J. Autism Dev. Disord 42 (2012) 1539-1548.

[25] G.C. Campione, C. Piazza, L. Villa, M. Molteni, Three-dimensional kinematic analysis of prehension movements in young children with autism spectrum disorder: new insights on motor impairment, J. Autism Dev. Disord. 46 (2016) 1985-1999.

[26] C.M. Glazebrook, D. Elliott, J. Lyons, A kinematic analysis of how young adults with and without autism plan and control goal-directed movements, Motor Contro 10 (2006) 244-264.

[27] C. Glazebrook, D. Gonzalez, S. Hansen, D. Elliott, The role of vision for online control of manual aiming movements in persons with autism spectrum disorders, Autism (2009), https://doi.org/10.1177/1362361309105659.

[28] M. Mari, U. Castiello, D. Marks, C. Marraffa, M Prior, The reach-to-grasp movemen in children with autism spectrum disorder, Philos. Trans. R. Soc. B Biol. Sci. 358 (2003) 393-403.

[29] S. Forti, et al., Motor planning and control in autism. A kinematic analysis of preschool children, Res. Autism Spectr. Disord. 5 (2011) 834-842.
[30] H.-.C. Yang, I.-.C. Lee, I.-.C Lee, Visual feedback and target size effects on reach-tograsp tasks in children with autism, J. Autism Dev. Disord. 44 (2014) 3129-3139.

[31] N. Nazarali, C.M. Glazebrook, D Elliott, Movement planning and reprogramming in individuals with autism, J. Autism Dev. Disord. 39 (2009) 1401-1411.

[32] Y. Fang, J. Wang, Y. Zhang, J. Qin, The relationship of motor coordination, visual perception, and executive function to the development of 4-6-year-old chinese preschoolers' visual motor integration skills, BioMed. Res. Int. 2017 (2017).

[33] C. von Hofsten, B. Rösblad, The integration of sensory information in the development of precise manual pointing, Neuropsychologia 26 (1988) 805-821.

[34] P. Bosco, et al., Brainstem enlargement in preschool children with autism: results from an intermethod agreement study of segmentation algorithms, Hum. Brain Mapp. 40 (2019) 7-19.

[35] J. Delafield-Butt, C. Trevarthen, On the brainstem origin of autism: disruption to movements of the primary self, Autism Mov. Sens. Perspect. (2017) 119-138.

[36] R.J. Jou, T.W. Frazier, M.S. Keshavan, N.J. Minshew, A.Y Hardan, A two-year longitudinal pilot MRI study of the brainstem in autism, Behav. Brain Res. 251 (2013) 163-167.

[37] R.J. Jou, N.J. Minshew, N.M. Melhem, M.S. Keshavan, A.Y Hardan, Brainstem volumetric alterations in children with autism, Psychol. Med. 39 (2009) 1347-1354.

[38] N.J. Rinehart, et al., An examination of movement kinematics in young people with high-functioning autism and asperger's disorder: further evidence for a motor planning deficit, J. Autism Dev. Disord. 36 (2006) 757-767.

[39] R.L. Shafer, Z. Wang, J. Bartolotti, M.W Mosconi, Visual and somatosensory feedback mechanisms of precision manual motor control in autism spectrum disorder, J. Neurodev. Disord. 13 (2021) 32.

[40] D. Wu, J.V. José, J.I. Nurnberger, E.B Torres, A biomarker characterizing neurodevelopment with applications in autism, Sci. Rep 8 (2018) 614.

[41] A.S. Mitchell, et al., A New Ipad Game For Ecological Motor Assessment of children: Bespoke Wearable and Smart Tablet Engineering For Autism-friendly assessment, International Society for Autism Research Annual Conference, Rotterdam, The Netherlands, 2018.

[42] J.T. Delafield-Butt, et al., Prospective organization of neonatal arm movements: a motor foundation of embodied agency, disrupted in premature birth, Dev. Sci. 21 (2018) e12693.

[43] J.M. Gottwald, S. Achermann, C. Marciszko, M. Lindskog, G. Gredebäck, An embodied account of early executive-function development: prospective motor control in infancy is related to inhibition and working memory, Psychol. Sci. 27 (2016) 1600-1610.

[44] D.N. Lee, General Tau Theory: evolution to date, Perception 38 (2009) 837-850.

[45] M.L. Gonzalez-Gadea, et al., Predictive coding in autism spectrum disorder and attention deficit hyperactivity disorder, J. Neurophysiol. 114 (2015) 2625-2636.

[46] S. Van de Cruys, et al., Precise minds in uncertain worlds: predictive coding in autism, Psychol. Rev. 121 (2014) 649-675.

[47] T. Liu, et al., Altered electroencephalogram complexity in autistic children shown by the multiscale entropy approach, Neuroreport 28 (2017) 169-173.

[48] S.H. Fatemi, et al., Consensus Paper: pathological Role of the cerebellum in autism, The Cerebellum 11 (2012) 777-807.

[49] J.P. Welsh, E.S. Ahn, D.G Placantonakis, Is autism due to brain desynchronization? Int. J. Dev. Neurosci. 23 (2005) 253-263.

[50] J.P. Welsh, E.J. Lang, I. Suglhara, R. Llinás, Dynamic organization of motor control within the olivocerebellar system, Nature 374 (1995) 453-457.

[51] P.R. Culmer, M.C. Levesley, M. Mon-Williams, J.H.G. Williams, A new tool for assessing human movement: the kinematic assessment tool, J. Neurosci. Methods 184 (2009) 184-192.

[52] A. Matic, A. Gomez-Marin, A customizable tablet app for hand movement research outside the lab, J. Neurosci. Methods 328 (2019), 108398.

[53] L. Millar, et al., Phase 3 diagnostic evaluation of a smart tablet serious game to identify autism in 760 children 3-5 years old in Sweden and the United Kingdom, BMJ Open 9 (2019), e026226. 